\title{
Effect of Malvidin on Induction of Apoptosis and Inhibition of Cell Proliferation on Myeloid and Lymphoid Leukemia
}

Haytham Dahlawi ${ }^{1 *}$

\author{
${ }^{1}$ Clinical Laboratory Sciences Department, College of Applied Medical Sciences, Taif University, P.O. Box 11099, Taif 21944, Saudi \\ Arabia
}

DOI: $10.36347 /$ sjams.2022.v10i01.025

| Received: 14.12.2021 | Accepted: 25.01.2022 | Published: 30.01.2022

*Corresponding author: Haytham Dahlawi

Clinical Laboratory Sciences Department, College of Applied Medical Sciences, Taif University, P.O. Box 11099, Taif 21944, Saudi Arabia

\section{Abstract}

Original Research Article

Malvidin is one of 6 most common anthocyanidins found in fruits and vegtables. studies suggest that anthocyanidins are bioactive chemicals have potential for treatment of cancer. Malvidin have been demonstrated to induce apoptosis and inhibition of cell proliferation in a number of cancer cell lines. However, to date, few studies have tested the potential of malvidin in the treatment of leukemia. In the present study, we investigated the antiproliferative and proapoptotic properties of malvidin in two human leukemia cell lines lymphoid cell line: SUP-B15 (acute lymphoblastic leukemia) and myeloid cell line: KG-1a (acute myelogenous leukemia) in addition to non-tumour control cells (CD133 ${ }^{+}$HSC). We found that treatment of cells with malvidin at concentration $0,25,50$ and $100 \mu \mathrm{M}$ for $24 \mathrm{~h}$ resulted in significant inhibition of cell proliferation in both leukemia cell lines with obvious less cytotoxic effect on non-tumour control cells. In addition, malvidin induce apoptosis in a dose and dependent manner in both leukemia cells and confirmed that by activation of caspas-3 following $24 \mathrm{~h}$ treatment. Cell arrest at $\mathrm{S}$ phase has been indicated in both cell lines using DNA analysis by flow cytometry. These finding indicated that malvidin could inhibit leukemic cell proliferation and induce their apoptosis and might be used as one kind of functional food component or a novel nutraceutical beneficial for blood health.

Keywords: Malvidin, anthocyanidins, treatment of cancer, fruits and vegtables, non-tumour control cells.

Copyright ( 0 2022 The Author(s): This is an open-access article distributed under the terms of the Creative Commons Attribution 4.0 International License (CC BY-NC 4.0) which permits unrestricted use, distribution, and reproduction in any medium for non-commercial use provided the original author and source are credited.

\section{INTRODUCTION}

Leukemia is described as a group of hematological diseases characterized by abnormal proliferation and growth of malignant hematopoietic cells within bone marrow or peripheral blood. These malignant cells can be classified as either myeloid or lymphoid depending on predominant cell type involved. In addition leukemia can be considered as either acute or chronic depending patient's age and on degree of cell differentiation. Globally in the last few years the number of new cases of leukemia significantly increased. Thus according to GLOBOCAN report leukemia is the fifteenth most frequently diagnosed cancer worldwide and the tenth main cause of cancer death [1]. It is the most common type of cancer in children and accounting for $30 \%$ of cases in children cancer [2]. Despite recent advance and availability different types of therapies to treat leukemia, however due to their specialization targeting a single target, they can induce toxicity and adverse effects.
Natural compounds derived from a range of sources may have the ability to stimulate a variety of physiological pathways which may be effective in the treatment of persistent disorders like cancer [3-5]. Numerous studies have been conducted to develop cancer treatments using natural compounds, particular phytochemical. Recent researches suggests that natural compounds derived from fruits can target a variety of leukemia-related pathways, providing positive feedback against malignancies and also playing a critical role in leukemia prevention [6-11].

Anthocyanidins are abundant throughout the plant kingdom. Anthocyanidins are found in abundance in red wine, purple cabbage, berries, and grapes [12]. Anthocyanidins have been shown to reduce the risk of cancer, liver, kidney, chronic inflammation and cardiovascular disease through their anti-mutagenesis, antioxidant, and anti-inflammatory properties [13, 3, 12]. According to a recent studies, the orthodihydroxyphenyl structure on ring number $\mathrm{B}$ is 
important for inhibiting tumour growth and metastasis $[14,15]$.

Malvidin is one of most commonly anthocyanidins found in vegetables and fruits. It is an abundant dietary anthocyanins found in blueberry and red grape, in 3-position glycosylated forms, exhibits anti-oxidant, anti-obesity, anti-inflammatory [16-19]. In term of tumour cancer, malvidin has shown to inhibit different tumour cell lines [20-22], and illustrated most potent antiproliferative among most common anthocyanins [22], therefore it has potential to be as anticancer agent. In leukemia only few studies examined the effect of malvidin on inhibition of leukemia cell lines.

Nonetheless, despite the fact that a number of researches have illustrated the anti-proliferative and induction of apoptosis characteristics of malvidin in different tumour cells, its effect on inhibition of cell proliferation and apoptosis induction on leukemia cells is not yet well studied. Therefore, the aim of this study is to investigate the effect of malvidin cytotoxicity on induction of apoptosis and inhibition cell proliferation on two different lineage of leukemia cell lines, in addition to explore it effect on non-tumour control cell.

\section{Material ANd Methods Cell culture}

In this study we used tow human leukemia cell lines lymphoid cell line: SUP-B15 (acute lymphoblastic leukemia) (ATCC: CRL1929) and myeloid cell line: KG-1a (acute myelogenous leukemia) (ATCC: CCL246.1), were purchases from the American Type Culture Collection (ATCC; Middlesex, U.K.). In addition, non-tumour control cells from cord blood $\left(\mathrm{CD}_{133^{+}}\right.$HSC) was obtained from Stem Cell Technologies (Grenoble, France). All cells were cultured in RPMI 1640 medium (Invitrogen, Paisley, U.K.). Media was supplied with $10 \%$ (v/v) fetal bovine serum, $1.5 \mathrm{mmol} / \mathrm{L}$ L-glutamine, and $100 \mathrm{lg} / \mathrm{mL}$ penicillin/streptomycin (Sigma). The cells were maintained at $37^{\circ} \mathrm{C}$ in a humidified incubator $0 \mathrm{f} 95 \%$ air and $5 \% \quad \mathrm{CO}_{2}$. The MycoAlert ${ }^{\mathrm{TM}}$ mycoplasma detection kit (Lonza Walkersville, Inc) was used in this study to test all cell for mycoplasma contamination.

Malvidin was dissolved in ethanol and used to treat leukemia cell lines in addition to non-tumour control cells for $24 \mathrm{~h}$ at concentration $(0,25,50,100$ $\mu \mathrm{M})$. All treatments in this study were performed in triplicate, in three independent experiments.

\section{Cell Viability}

Cell viability was quantified by CellTiterGlo ${ }^{\circledR}$ Luminescent Cell Viability Assay Kit (Promega, Southampton, UK) as homogenous method based on quantification of ATP levels to determine metabolic active cells. In brief, leukemia cell lines (SUP-B15 and KG-1a) in addition to non-tumour control cells
$\left(\mathrm{CD} 133^{+}\right.$HSC) were seeded into white 96-well plated (Fisher Scientific, Loughborough, UK) at density $2.5 \mathrm{x}$ $10^{3}$ cells per well and treated as indicated concentration of malvidin for $24 \mathrm{~h}$. Background luminescence was determined by preparing control wells containing only medium or treatments without cells. Following $24 \mathrm{~h}$ incubation, the 96-well plate was equilibrated for 30 minutes at room temperature. Then, Cell Titer-Glo Reagent $(100 \mu \mathrm{l})$ was added to each well, mixed well for 2 minutes prior to detect luminescence using synergy neo2 luminescence detector (BioTek, Oxfordshire, UK). The IC50 was determined for malvidin in each cell line.

\section{Assessment of Apoptosis by Annexin V/Propidium Iodide Staining}

The effect of malvidin on induction apoptosis was examined using annexin V-FITC and PI. Leukemia cells (SuB-15 and KG-1a) were seeded in 12-well plates at cell density $0.5 \times 10^{6}$ per well and treated with malvidin at indicated concentration for $24 \mathrm{~h}$. Following treatment, cells were centrifuged at $400 \mathrm{~g}$ for 5 minutes and washed two times with cold PBS. Then cells were resuspended in binding buffer containing $1 \mathrm{mg} / \mathrm{mL}$ propidium iodide (PI) and $1 \mathrm{mg} / \mathrm{mL}$ FITC-labeled Annexin-V. After that, cells were incubated for 5 minutes in dark on ice. Following staining, all samples were analysed by BD FACS Calibur flow cytometer. For each sample, 10,000 cells was recorded and data analysed using FlowJo software (Tree Star, Ashland, OR).

\section{Assessment of Caspase-3 Activity}

Caspase 3, play an important role in the initiation of cellular events during early apoptosis. The caspase 3 activity was determined using NucView caspase 3 activity substrate (Cambridge Bioscience, Cambridge, U.K.) based on flow cytometry. Cells were seeded in 12-well plates (Fisher Scientific) and then treated with malvidin as indicated concentration for 24 h. After that, $200 \mu \mathrm{l}$ of cell suspension was transferred to tubes of flow cytometry and $5 \mu$ l of caspase 3 activity assay $(0.2 \mathrm{mM})$ was added directly and mixed well, then incubated for 10 minutes in dark at room temperature. Prior to analysis using FACS Calibur Cytometer (BectonDickinson, U.K.), $300 \mu \mathrm{l}$ of Dulbecco's phosphate-buffered saline (DPBS) (Invitrogen, Paisley, U.K.) was added. For each sample, 10,000 cells was recorded and data analysed using FlowJo software (Tree Star, Ashland, OR).

\section{DNA Cell Cycle Analyisi:}

The effect of malvidin on cell cycle progression was investigated using Propidium iodide (PI) satin based on flow cytometry. Sub-B15 and KG1a cells were seeded in 12 well plates at $0.5 \times 10^{6}$ cells per well and treated with malvidin at indicated concentration for $24 \mathrm{~h}$. Following treatment, cells were trypsinised and centrifuged at $400 \mathrm{~g}$ for 5 minutes. The supernatant was removed prior to wash cells twice with $200 \mu \mathrm{l}$ cold PBS and then fixed with $100 \mu \mathrm{l}$ of $80 \%$ 
ethanol/H2O (v/v) and incubated at $4 \mathrm{C}^{\circ}$ for at least $2 \mathrm{~h}$. Then fixed cells were washed twice with cold PBS before adding $50 \mu \mathrm{g} / \mathrm{mL}$ PI stain (Sigma, Poole UK) and $50 \mu \mathrm{l}$ of $0.1 \mathrm{unit} / \mathrm{mL}$ RNase (Sigma, Poole UK). After that, BD FACS Calibur flow cytometer was used to analyse each samples. For each sample, 10,000 cells was recorded and FlowJo software (Tree Star, Ashland, OR) using the Waston (pragmatic) equation (Tree Star, Ashland, OR,USA) was used to analyse DNA histogram of cell cycle.

\section{Statistical Analysis}

For each assay means and standard error of the mean (SEM) were calculated. SPSS software was used to indicate whether data parametric or no-parametric. As data were non-parametric a Kruskal-Wallis and Conover-Inman post hoc tests were used to investigates statistical significance of the data. Results were considered statistically significant when $P \leqslant 0.05$.

\section{RESUlTS}

To investigate whether malvidin effect the growth of leukemia cell lines (SUP-B15 and KG-1a) in addition to non-tumour cell lines (CD133), cells were treated with $0,25,50,100 \mu \mathrm{M}$ malvidin, after that cell viability was examined by CellTiter-Glo ${ }^{\circledR}$ Luminescent Cell Viability Assay. As shown in Figure 1 both SUPB15 and KG-1a cell viability significantly supressed after treating with malvidin when compared to negative control. After $24 \mathrm{~h}$, malvidin demonstrated high inhibition of cell proliferation growth in a dosedependent manner with $\mathrm{IC}_{50}$ values of $49 \mu \mathrm{M}$ (SUPB15) and $72 \mu \mathrm{M}$ (KG-1a). The lymphoid cell line was more sensitive to malvidin treatment than myeloid cell lines. The non-tumour cell line (CD133) less sensitive than leukaemia cell lines SUP-B15 and KG-1a at all treatment concentration following $24 \mathrm{~h}$ treatment with malvidin. Interestingly, malvidin failed to cause a $50 \%$ decrease in ATP in non-tumour cell line (CD133).

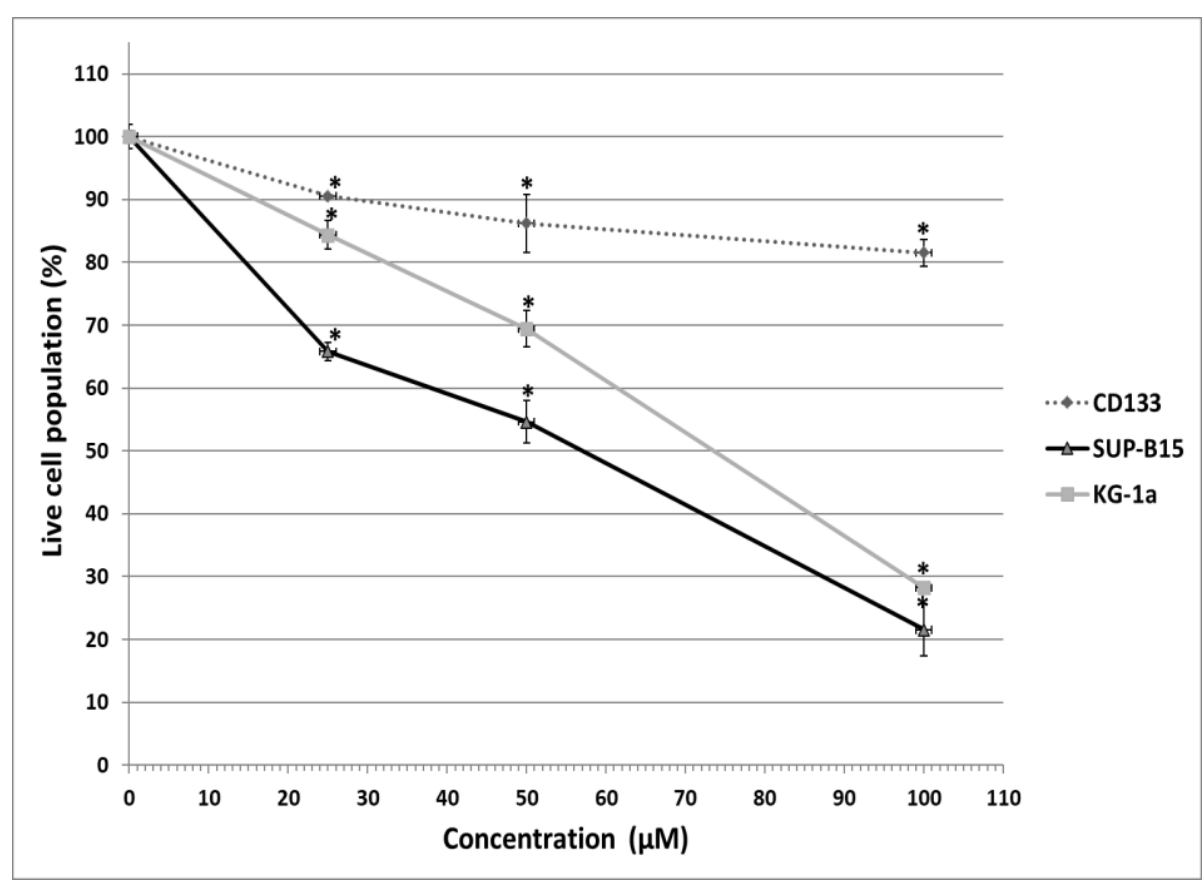

Figure 1: Cytotoxic effect of malvidin on induction of cell proliferation on myeloid leukemia cell line (KG-1a), lymphoid leukemia cell line (SUP-B15) and non-tumor control cell (CD133) using Cell Titer-Glo® Luminescent Cell Viability Assay to indicate live cell number. Cells treated at concentration (0, 25, 50 and $100 \mu \mathrm{M})$ for $24 \mathrm{~h}$. ATP level were normalized to control. Significant difference $(P \leq 0.05)$ versus untreated control is indicated by $(*)$

The effect of malvidin on induction of apoptosis was investigated by flow cytometry using annexin V-FITC and PI. As shown in Figure 2 malvidin demonstrated in both leukemia cell lines SUP-B15 and KG-1a following $24 \mathrm{~h}$ treatment significant $(P \leqslant 0.05)$ increase in the apoptotic cell number and dead cells and significant $(P \leqslant 0.05)$ decrease in the number of live cells in a dose-dependent manner with different sensitivity between these two cell lines. 


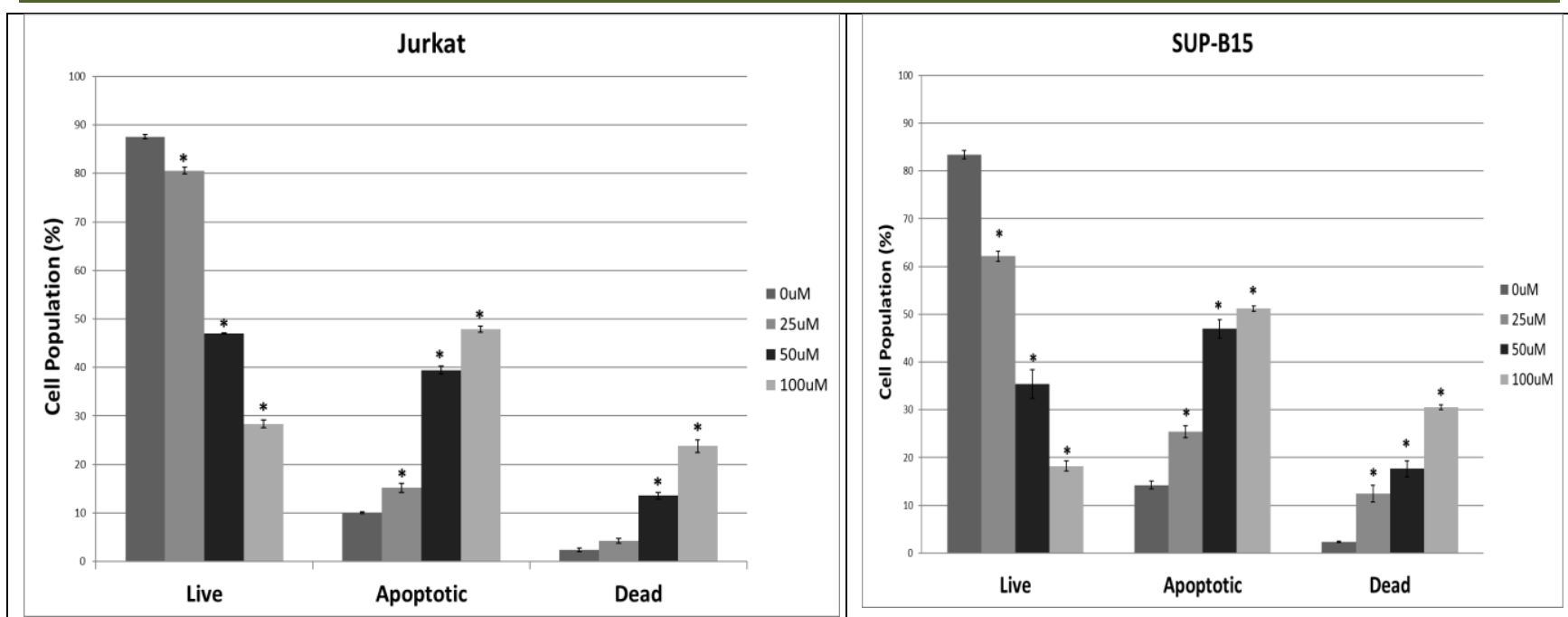

Figure 2: Cytotoxic effect of malvidin on induction apoptosis on myeloid leukemia cell line (KG-1a), lymphoid leukemia cell line (SUP-B15) using Annexin V-FITC/ PI based on flow cytometry. Cells treated at concentration ( $0,25,50$ and $100 \mu \mathrm{M})$ for $24 \mathrm{~h}$. Significant difference $(P \leq 0.05)$ versus untreated control is indicated by (*)

We further investigated the molecular events underlying the malvidin induced apoptosis in leukemia cell lines SUP-B15 and KG-1a using NucView caspase 3 activity substrate base on flow cytometry. To assess the involvement of caspase-3, we investigated its activities in the malvidin treated and non-treated control
SUP-B15 and KG-1a at concentration $0,25,50$, and $100 \mu \mathrm{M}$ following $24 \mathrm{~h}$ treatment. As shown in Figure 3 caspse-3 activity in the malvidin treated cells significantly $(P \leqslant 0.05)$ increased than in that control cells in both leukemia cell lines.

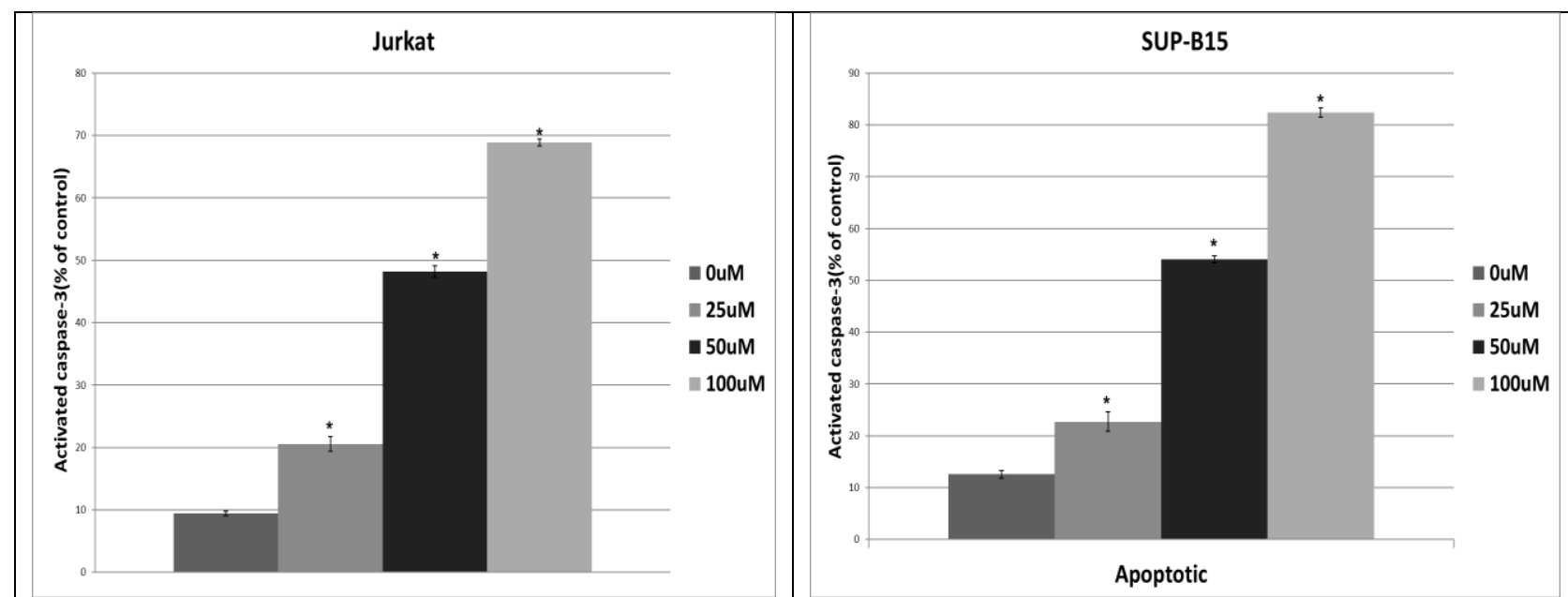

Figure 3: Effect of malvidin on caspase-3 activation on myeloid leukemia cell line (KG-1a), lymphoid leukemia cell line SUP-B15) using NucView ${ }^{\mathrm{TM}} 488$ Caspase-3 substrate based on flow cytometry. Cells treated at concentration $(0,25,50$ and $100 \mu \mathrm{M})$ for $24 \mathrm{~h}$. significant difference $(P \leq 0.05)$ versus untreated control is indicated by $(*)$. Mean \pm SEM.

To examine the effect of malvidin treatment decrease the percentage of live cells via cell cycle arrest and /or apoptosis, the distribution of cells in different phases of cell cycle was investigated using DNA cell cycle analysis based on flow cytometry. Cell number in each phase (G0/G1, S and $\mathrm{G} 2 / \mathrm{M}$ ) is expresses as percentage of the total number of cells analysed. The myeloid cell lines KG-1a illustrated a significant $(P \leqslant 0.05)$ accumulation of cells in $\mathrm{S}$ phase at only concentration 50 and $100 \mu \mathrm{M}$ and decrease in G0/G1 phase following treatment with malvidin for $24 \mathrm{~h}$. However, malvidin cause significant $(P \leqslant 0.05)$ accumulation of cells in $\mathrm{S}$ phase corresponding with a decrease in $\mathrm{G} 0 / \mathrm{G} 1$ and $\mathrm{M}$ phase at all concentration following treatment for $24 \mathrm{~h}$ in lymphoid cell lines SUP-B15 Figure 4. 


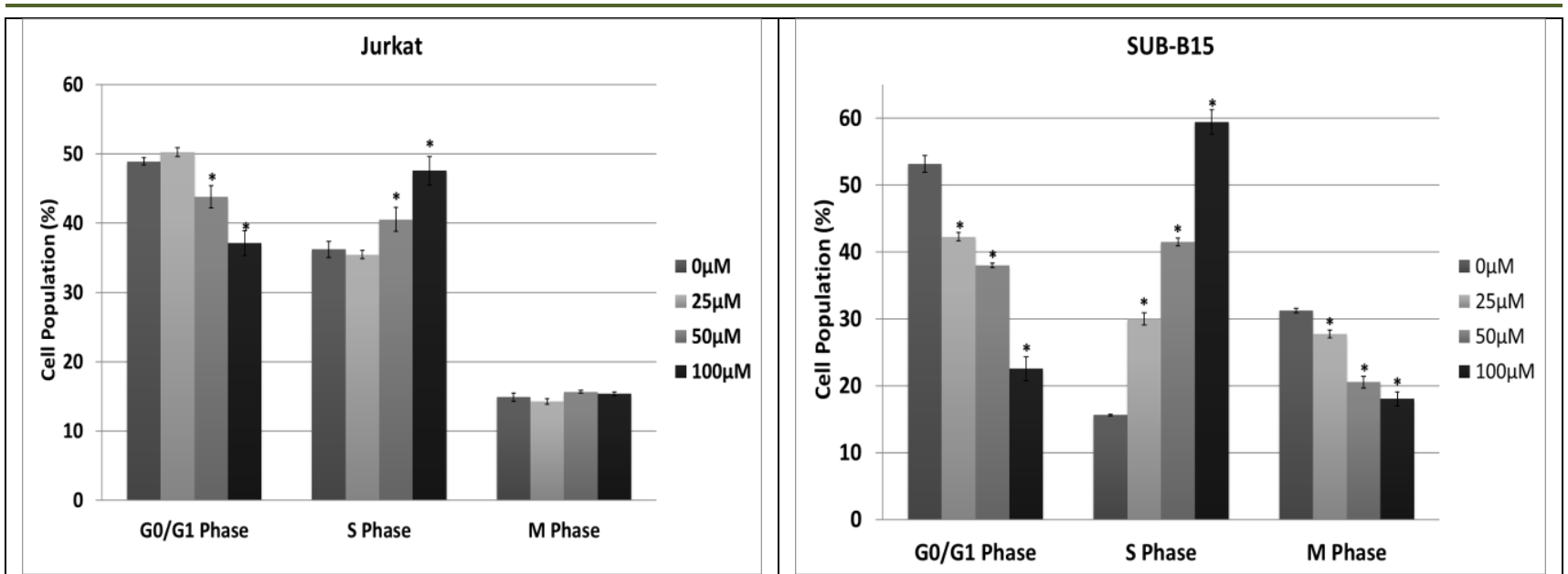

Figure 4: Effect of malvidin on cell cycle arrest on myeloid leukemia cell line (KG-1a) and lymphoid leukemia cell line (SUP-B15) using PI satin based on flow cytometry, following treatment with malvidin at concentration $(0,25,50$ and $100 \mu \mathrm{l})$ for 24 . Significant difference $(P \leq 0.05)$ versus untreated control is indicated by $(*)$

\section{DISCUSSION}

Despite of many breakthrough in leukemia treatment, leukemia is still one of the most major causes of death worldwide. Natural compounds, such as anthocyanins found in abundance in human diet particularly fruits, have been found to reduce risk of cancer by induction of apoptosis and inhibition of cell proliferation [23-25, 3].

The hallmark of leukaemia is an increase in cellular proliferation rate of leukemic cells. The current study demonstrated that malvidin inhibits cell proliferation in both lymphoid leukemia cell line (SUPB15) and myeloid leukemia cell line (KG-1a). Few studies have been tested the effect of malvidin on leukaemia cell lines. Katsube and colleagues studied the effect of 5 pure anthocyanidins on inhibition of cell proliferation in leukemia cell line (HL-60). Malvidin demonstrated the most potent antiproliferation in HL-60 among other anthocyanidins [26]. In addition, malvidin extracted from Oryza sativa showed growth inhibition on U937, human monocytic leukemia cells [27]. In cancer cell lines, of five anthocyanidins (cyanidin, delphinidin, malvidin, pelargonidin, and peonidin) malvidin demonstrated the greatest cell proliferation inhibition on human caucasian gastric adenocarcinomm [26]. Furthermore, Malvidin demonstrated the most potent inhibition among cyanidin, delphinidin, pelargonidin and petunidin on HCT116, NCIH460, SF268 and MCF7 cancer cell proliferation [28]. Interestingly, this study showed that non-tumour control cells $\left(\mathrm{CD}_{133^{+}}\right.$HSC) less sensitive than lymphoid leukemia cell line (SUP-B15) and myeloid leukemia cell line (KG-1a) at all doses following $24 \mathrm{~h}$ treatment, suggesting selective antiproliferation effect against leukemia cells. Selective cytotoxicity in cancer cells of anthocyanins has been studied previously. Zhao et al., (2004) reported that anthocyanins inhibited cell proliferation of colon cancer cells (HT-29) with little cytotoxic effect on normal colonic cell proliferation.
Also Wang et al (2019) reported no cytotoxic effect on normal liver cell lines while having a significant cytotoxicity against human hepatoma HepG2 cells.

In addition to uncontrolled cell growth, resistance to apoptosis is considered another important hallmark of leukemia cells. Induction of apoptosis by malvidin have been seen within few leukemia cell lines. In 2004, study reported induction of apoptosis on U937, human monocytic leukemia cells by malvidin extracted from Oryza sativa [27]. Kobori (2003) reported induction of apoptosis in HL-60 leukemia cell lines but not in HCT116 human colon carcinoma cells. Wang et al., (2019) reported that malvidin-3-galactoside induced Human hepatoma HepG2 cells apoptosis [29]. Similarly, Dhivya et al, reported malvidin induce apoptosis in Human hepatoma HepG2 cells [30]. In this study we demonstrated malvidin induce apoptosis on both leukemia cell lines SUP-B15 and KG-1 showing a significant decrease in live cell with a significant increase in apoptotic and dead cells, indicating different sensitivity between cell lines.

In this study we confirmed the occurrence of apoptosis induced by malvidin through caspase-3 activation. Capase-3 play an important roles in initiating the caspase cascade reaction. We reported significant activation of capase-3 at all doses with different sensitivity in both cell lines (SUP-B15 and KG-1a). Similarly, In human gastric adenocarcinoma AGS cells, induction of apoptosis by malvidin was confirmed by different mechanisms including caspase-3 activation [22]. Y. Ma et al., (2020) reported malvidin activate caspase-3 in hepatic stellate T6 cells (HSCT6) following $24 \mathrm{~h}$ treatment [31]. Lopes et al., (2015) showed that malvidin failed to activate caspae-3 in human astrocytoma U373 MG line compared to control. However the treatment incubation period was for short time (45 minutes) comparing to our study [32]. 
Haytham Dahlawi; Sch J App Med Sci, Jan, 2022; 10(1): 150-156

Both cell growth inhibition and apoptosis induction are highly correlated with the activation of a number of intracellular signalling pathways that lead to cell cycle arrest in the G1, S, or G2/M phase [33]. This study showed arrest of cell cycle at $\mathrm{S}$ phase in both leukemia cell lines (SUP-B15 and KG-1a) with different sensitivity. Similarly, J. Lin et al., (2020) reported induction of cell cycle arrest at $\mathrm{S}$ phase following treatment with Malvidin-3-galactoside on Human HCC cell line Huh-7 [21]. Whilst G2/M phase arrest has been shown in human monocytic leukemia cells (U937) [27].

\section{CONCLUSION}

Our results demonstrated that malvidin inhibit cell proliferation of leukemia cell lines, and showed variation in sensitivity between lymphoid and myeloid cell lines and non-tumour control cells. Furthermore, our results demonstrated induction of apoptosis eident in SUP-B15 and KG-1a leukemia cell lines. Also, arrest at $\mathrm{S}$ phase of cell cycle was indicated following treatment with malvidin. He current study suggests that malvidin has potential for therapeutic application in treatment of leukemia, and further work is required to confirm these finding.

\section{REFERENCES}

1. Sung, H., Ferlay, J., Siegel, R. L., Laversanne, M., Soerjomataram, I., Jemal, A., \& Bray, F. (2021). Global cancer statistics 2020: GLOBOCAN estimates of incidence and mortality worldwide for 36 cancers in 185 countries. CA: a cancer journal for clinicians, 71(3), 209-249.

2. Kaplan, J. A. (2019). Leukemia in children. Pediatr Rev, 40(7), 319-331.

3. Hazafa, A., Rehman, K. U., Jahan, N., \& Jabeen, Z. (2020). The role of polyphenol (flavonoids) compounds in the treatment of cancer cells. Nutrition and cancer, 72(3), 386-397.

4. Subramaniam, S., Selvaduray, K. R., \& Radhakrishnan, A. K. (2019). Bioactive compounds: natural defense against cancer?. Biomolecules, 9(12), 758.

5. Lin, S. R., Chang, C. H., Hsu, C. F., Tsai, M. J., Cheng, H., Leong, M. K., ... \& Weng, C. F. (2020). Natural compounds as potential adjuvants to cancer therapy: Preclinical evidence. British journal of pharmacology, 177(6), 1409-1423.

6. Morceau, F., Chateauvieux, S., Orsini, M., Trécul, A., Dicato, M., \& Diederich, M. (2015). Natural compounds and pharmaceuticals reprogram leukemia cell differentiation pathways. Biotechnology advances, 33(6), 785797.

7. Hwang, D., Kim, M., Park, H., Jeong, M. I., Jung, W., \& Kim, B. (2019). Natural products and acute myeloid leukemia: A review highlighting mechanisms of action. Nutrients, 11(5), 1010.

8. Saraei, R., Marofi, F., Naimi, A., Talebi, M.,
Ghaebi, M., Javan, N., ... \& Hassanzadeh, A. (2019). Leukemia therapy by flavonoids: Future and involved mechanisms. Journal of cellular physiology, 234(6), 8203-8220.

9. Taverna, S., \& Corrado, C. (2017). Natural compounds: molecular weapons against Leukemia's. J Leuk, 5(226), 2.

10. Salimi, A., \& Pourahmad, J. (2018). Role of natural compounds in prevention and treatment of chronic lymphocytic leukemia. Polyphenols: prevention and treatment of human disease, 195-203.

11. Maher, T., Ahmad Raus, R., Daddiouaissa, D., Ahmad, F., Adzhar, N. S., Latif, E. S., ... \& Mohammed, A. (2021). Medicinal Plants with Anti-Leukemic Effects:

Review. Molecules, 26(9), 2741.

12. Speer, H., D’Cunha, N. M., Alexopoulos, N. I., McKune, A. J., \& Naumovski, N. (2020). Anthocyanins and human health - a focus on oxidative stress, inflammation and disease. Antioxidants, 9(5), 366.

13. Blesso, C. N. (2019). Dietary anthocyanins and human health. Nutrients, 11(9), 2107.

14. Chen, J., Xu, B., Sun, J., Jiang, X., \& Bai, W. (2021). Anthocyanin supplement as a dietary strategy in cancer prevention and management: A comprehensive review. Critical Reviews in Food Science and Nutrition, 1-13.

15. Stoner, G. D., Wang, L. S., \& Casto, B. C. (2008). Laboratory and clinical studies of cancer chemoprevention by antioxidants in berries. Carcinogenesis, 29(9), 1665-1674.

16. Hossain, R., Islam, M. T., Mubarak, M. S., Jain, D., Khan, R., \& Saikat, A. S. (2021). Natural-Derived Molecules as a Potential Adjuvant in Chemotherapy: Normal Cell Protectors and Cancer Cell Sensitizers. Anti-cancer Agents in Medicinal Chemistry, 21.

17. Abdin, M., Hamed, Y. S., Akhtar, H. M. S., Chen, D., Chen, G., Wan, P., \& Zeng, X. (2020). Antioxidant and anti-inflammatory activities of target anthocyanins di-glucosides isolated from Syzygium cumini pulp by high speed countercurrent chromatography. Journal of food biochemistry, 44(6), 1050-1062.

18. Lee, C., Han, D., Kim, B., Baek, N., \& Baik, B. K. (2013). Antioxidant and anti-hypertensive activity of anthocyanin-rich extracts from hulless pigmented barley cultivars. International journal of food science \& technology, 48(5), 984-991.

19. Saulite, L., Jekabsons, K., Klavins, M., Muceniece, R., \& Riekstina, U. (2019). Effects of malvidin, cyanidin and delphinidin on human adipose mesenchymal stem cell differentiation into adipocytes, chondrocytes and osteocytes. Phytomedicine, 53, 86-95.

20. Baba, A. B., Nivetha, R., Chattopadhyay, I., \& Nagini, S. (2017). Blueberry and malvidin inhibit cell cycle progression and induce mitochondrialmediated apoptosis by abrogating the JAK/STAT-3 
Haytham Dahlawi; Sch J App Med Sci, Jan, 2022; 10(1): 150-156

signalling pathway. Food and Chemical Toxicology, 109, 534-543.

21. Lin, J., Tian, J., Shu, C., Cheng, Z., Liu, Y., Wang, W., ... \& Wang, Y. (2020). Malvidin-3-galactoside from blueberry suppresses the growth and metastasis potential of hepatocellular carcinoma cell Huh-7 by regulating apoptosis and metastases pathways. Food Science and Human Wellness, 9(2), 136-145.

22. Shih, P. H., Yeh, C. T., \& Yen, G. C. (2005). Effects of anthocyanidin on the inhibition of proliferation and induction of apoptosis in human gastric adenocarcinoma cells. Food and chemical toxicology, 43(10), 1557-1566.

23. Shen, Y., Zhang, N., Tian, J., Xin, G., Liu, L., Sun, X., \& Li, B. (2022). Advanced approaches for improving bioavailability and controlled release of anthocyanins. Journal of Controlled Release, 341, 285-299.

24. Ma, J., Song, L., \& Zhao, C. (2022). Purple sweet potato anthocyanin regulates the proliferation, migration and invasion of breast cancer MDA-MB231 cells through circ_0003998/miR-145 axis. Chinese J Cancer Biother, 28.

25. Dharmawansa, K. V., Hoskin, D. W., \& Rupasinghe, H. P. (2020). Chemopreventive effect of dietary anthocyanins against gastrointestinal cancers: A review of recent advances and perspectives. International Journal of Molecular Sciences, 21(18), 6555.

26. Katsube, N., Iwashita, K., Tsushida, T., Yamaki, K., \& Kobori, M. (2003). Induction of apoptosis in cancer cells by bilberry (Vaccinium myrtillus) and the anthocyanins. Journal of agricultural and food chemistry, 51(1), 68-75.

27. Hyun, J. W., \& Chung, H. S. (2004). Cyanidin and malvidin from Oryza sativa cv. Heugjinjubyeo mediate cytotoxicity against human monocytic leukemia cells by arrest of G2/M phase and induction of apoptosis. Journal of agricultural and food chemistry, 52(8), 2213-2217.

28. Zhang, Y., Vareed, S. K., \& Nair, M. G. (2005). Human tumor cell growth inhibition by nontoxic anthocyanidins, the pigments in fruits and vegetables. Life sciences, 76(13), 1465-1472.

29. Wang, Y., Lin, J., Tian, J., Si, X., Jiao, X., Zhang, W., ... \& Li, B. (2018). Blueberry malvidin-3galactoside suppresses hepatocellular carcinoma by regulating apoptosis, proliferation, and metastasis pathways in vivo and in vitro. Journal of agricultural and food chemistry, 67(2), 625-636.

30. Dhivya, S., Khandelwal, N., Abraham, S. K., \& Premkumar, K. (2016). Impact of anthocyanidins on mitoxantrone-induced cytotoxicity and genotoxicity: an in vitro and in vivo analysis. Integrative cancer therapies, 15(4), 525534.

31. Ma, Y., Li, Y., Zhang, H., Wang, Y., Wu, C., \& Huang, W. (2020). Malvidin induces hepatic stellate cell apoptosis via the endoplasmic reticulum stress pathway and mitochondrial pathway. Food Science \& Nutrition, 8(9), 50955106.

32. López, A., Palomino, O., Ortega, T., \& Carretero, E. (2015). Protective Effect of Anthocyanidins on Astrocytes and Apoptosis Induced by Oxidative Damage. Planta Medica Letters, 2(01), e19-e24.

33. Singh, R. P., Dhanalakshmi, S., \& Agarwal, R. (2002). Phytochemicals as cell cycle modulators a less toxic approach in halting human cancers. Cell cycle, 1(3), 155-160. 